\title{
Analysis and Design of University Teaching Evaluation System Based on JSP Platform
}

\author{
Ning Huang ${ }^{a^{*}}$ \\ ${ }^{a}$ Center of Modern Educational Technology, Gannan Normal University, Ganzhou,341000,China
}

\begin{abstract}
High quality of teaching is fundamental purpose and basic task of a university, as well as a foothold in the university. We introduce in this paper a university teaching evaluation. This system is used by students and experts via Servlet+JavaBean+ORACLE on campus network with the foundation of the system published by the teaching affairs bureau of university. The target system is divided into student evaluation, expert evaluation and management modules. The evaluation system is divided into two subsystems, namely, expert evaluation and student evaluation of courses. Database is the core of the whole system. It serves all the information processing modules. The implementation of the system can fully improve the quality control of teaching and lower the cost. A teaching evaluation system is analyzed and designed in this paper.
\end{abstract}

Index Terms: Teaching evaluation, University, Network, Student, Teacher.

(C) 2017 Published by MECS Publisher. Selection and/or peer review under responsibility of the Research Association of Modern Education and Computer Science.

\section{Introduction}

High quality of teaching is both fundamental purpose and basic task of a university, meanwhile a foothold in the university. In the universities, we should establish a long-term idea of "quality for survival, quality for development" strictly to supervise and control teaching quality. We should continuously provide talents for the society by taking the observation and evaluation of teaching as an important part.

The traditional observation and evaluation methods used to distribute the paper questionnaire for students, instructor experts to evaluate sub-items. Students are asked to fill in a form of evaluation. So do the experts. When the forms are completed, collected, statistical analysis can be done. This approach is time-consuming, laborious, error-prone, impossible to be fast and correct. The disadvantage is obvious. And because the amount of data is quite limited, it is not practical to be widely used.

For online courses students and teacher are partners in the learning process. It is the same in the case of

* Corresponding author.

E-mail address: 
teaching evaluation. Students take an important role in the teaching quality evaluation. On the network, we can develop teaching evaluation system and promote university teaching quality control fully and effectively. Thus, evaluation system of teaching quality has been brought about. Online systems are used more and more. Searching online evaluation get a lot of results[1-6].

The rest of the paper is organized as follows. Section 2 focuses on main frame of the system; Section 3 focuses on overall design of target system; Section 4 focuses on design of the operation mode; Section 5 focuses on database design; Section 6 focuses on design of evaluation standardization; Section 7 focuses on effect analysis of evaluation system; Section 8 gives conclusions.

\section{Main Frame of the System}

The main frame of the system is illustrated in Fig.1(a). It is an important work to decide a score for each of the indexes, because the goal and direction is the foundation of constructing evaluation system[9]. Evaluation system of teaching quality in accordance with the standards bases on the factors of affecting the teaching quality in teaching, each link for systematically monitoring and evaluating system. The ultimate goal is to guarantee the quality of teaching in accordance with established quality standards. according to the principles of total quality management concepts and systems engineering.

The system includes three types of users, they are students, teachers and experts. The structure is shown in Fig.1(a) and Fig.1(b): Teaching evaluation system is illustrated in Fig.1(a). Types of teaching evaluation system are illustrated in Fig.1(b).

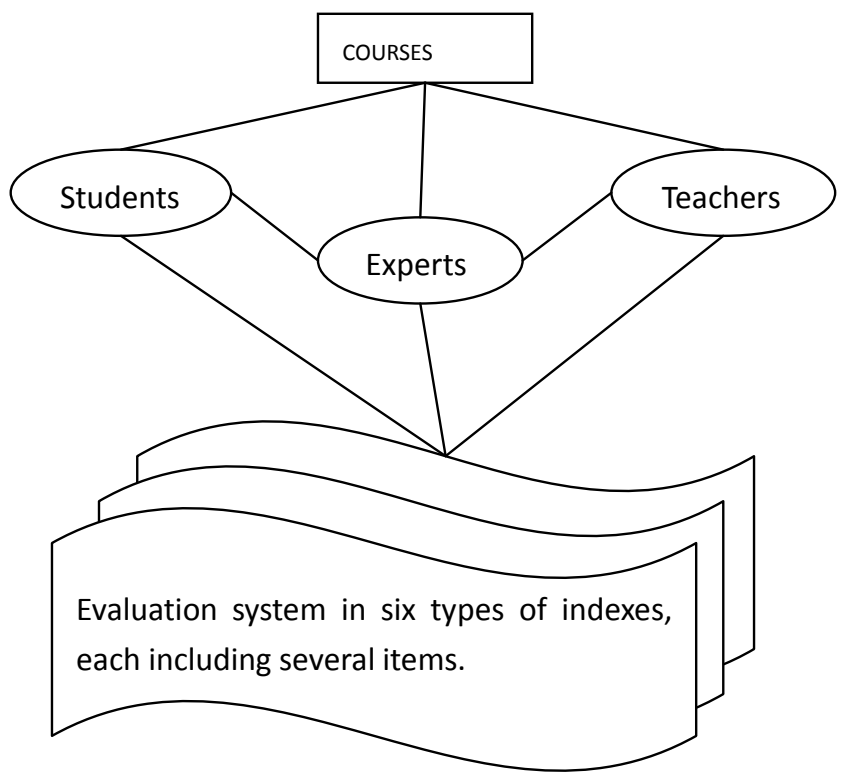

Fig.1(a). Teaching Evaluation System 


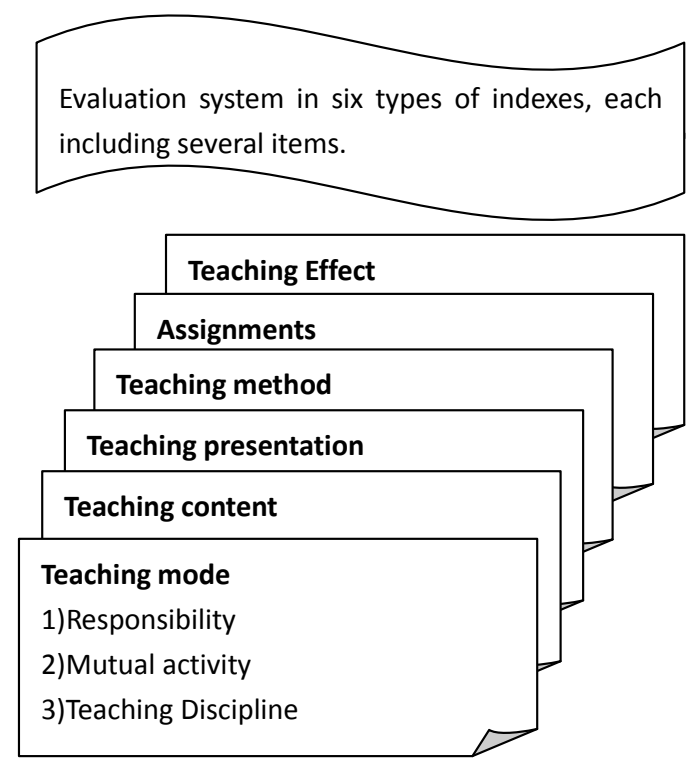

Fig.1(b). Types of Teaching Evaluation System

The evaluation system is divided into two subsystems, namely, expert evaluation and student evaluation of courses in two parts. These two subsystems make up the monitoring and evaluation system of teaching quality, with a strong scientific, systematic and operational characteristics, and the system is relatively independent and interrelated.

In this system, every student should give a score to the teacher at the end of the course. In addition, an education expert may supervise the teaching process and give the teacher a score.

Each score should be put into a database to facilitate departments to grasp the quality of teaching.

Teaching affairs bureau decides which index should be used to evaluate teaching quality.

After the system is running, by functional departments, experts, depending on the situation, analyzes and evaluates the reasonableness of each index in the system, amendments, approved by the dean's office, the system amending the assessment system.

\section{Overall Design of target System}

Target system is divided into three modules: student evaluation, expert evaluation and management evaluation modules. They should be called according to the authentication of the identity. The overall framework of the target system is shown in Fig. 2.

Here is a case of students' evaluation of courses and students sign on. They can see the list of courses and instructions. A student chooses either of these items; see a form "You are evaluating XX course of XX (teacher)" which is prescribed by the dean's office. Students can fill out the form before submission. All teacher and courses related to the student can be seen and evaluated.

Expert evaluation module is similar to that of the students. The assessment projects and methods of operation are almost the same, only different from each other in scoring weights.

Functional management module includes an administrator module to maintain administrators and users of teaching functional departments. It has the highest authority in this system, including adding, changing, deleting students, teachers, courses and classes. "Course" is the key link in the whole target system, as well as in the database. It is the tie between experts and teachers, the tie between students and teachers. Without this 
link, the valuation cannot be performed. Administrator module can generate evaluation results according to original data from student and expert evaluation, but it cannot modify the evaluation results produced by the original data. It is not allowed to modify the generated results either.

Students, experts, administrators can change their own password which is demanded abide by strong password guidelines.

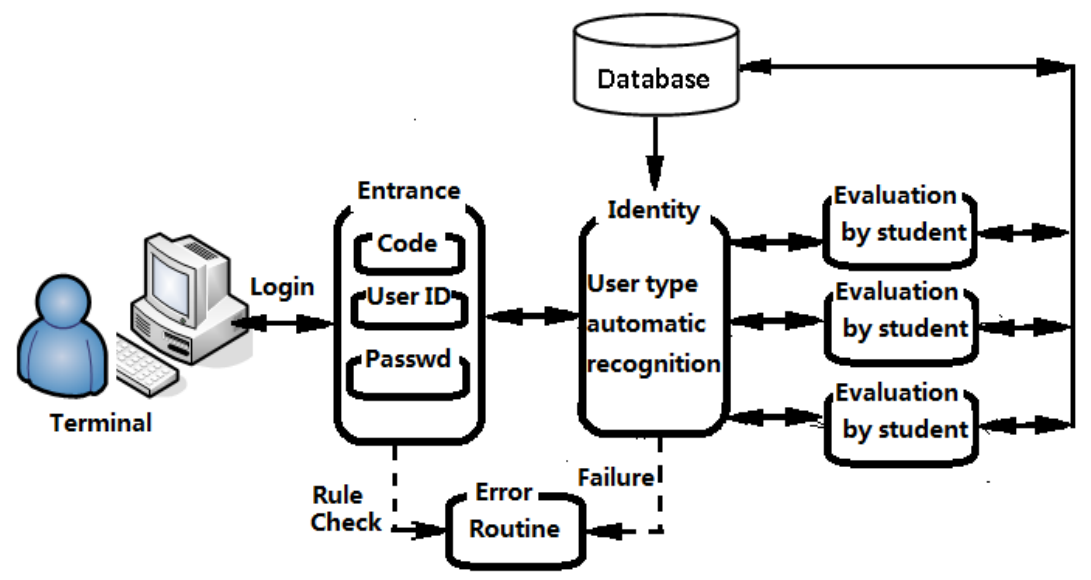

Fig.2.Target System Structure

\section{Design of the Operation Mode}

This system was designed with Servlet,JSP,JavaBean[1, 13] and Oracle. Servlet serves as main logic control. It accepts user requests coming from the JSP issue. It calls and initializes JavaBean. It is delivered to the client via JSP. SqlBean serves as the main connection between database and communication[14]. JavaBean assumes JSP and Servlet to complete the user's requests. Figure 3. Shows the running modes of teaching evaluation system.

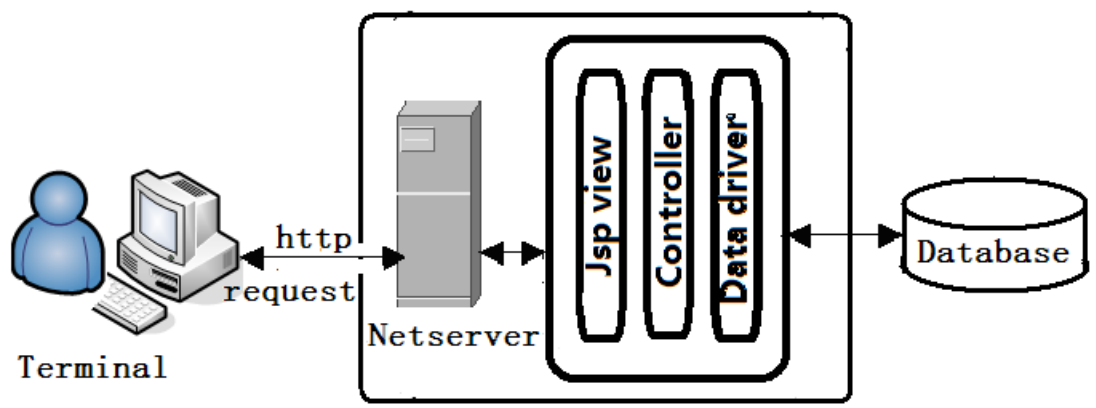

Fig.3. Operation mode of Evaluation System

This technique has the features of cross-platform, good portability and easy to use. For example, JSP code used in WINDOWS can be used in UNIX environments without any modifications. Java is a kind of system platform-independent computer language that is robust, secure and ease to use and get from the Internet. It is able to "write once, run anywhere" [15]. Thus, we can write a program under WINDOWS with a native work 
station and port code to UNIX server with higher stability and security. Communication with database uses SqlBean connection rather than directly accessing the database. UNIX is more stable and does not go down as often as WINDOWS does, therefore requires less administration and maintenance. UNIX has greater built-in security and many more advantages. It is the first choice as server system for many programmer[2]. But WINDOWS has more convenient interface, it is the proper desktop system.

The scheme is regardless of the details of each database dialect, which adapts to any kind of major database(Oracle,DB2,Informix,SQL Server, etc.).

This also provides for code migration. The popular B/S (Browser/Server) mode is used to design user interface to avoid install client software. Students and experts evaluate teaching quality via web pages. They $\log$ on to the system, select the object of evaluation which is given in the form of scoring, then submit the form. Administrator also enters the system by using a browser to open a web page.

\section{Database Design}

Database is the core of the whole system. It serves all the information processing modules. So, it should be focused on. When evaluating, a student selects a teacher from the list of teachers from database. The score should be stored into the database, to name a few.

Database includes some data tables. For example, in the affairs of evaluation by the students, the following tables are required:

1) Students list, including studen number, student name and class ID, etc.;

2) Curriculum, including teacher, course, and class ID etc.;

3) Teacher's score, including teacher name, course and score, etc. It is used to save score submitted by students. It also provides functions management sector for statistics and analysis of the information. Figure 4. shows association between tables;

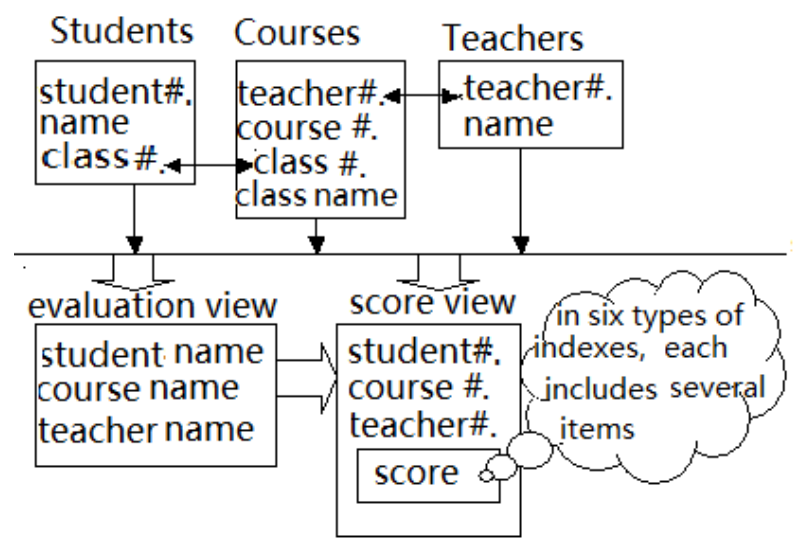

Fig.4. Correlation between Database Tables

In the affairs of evaluation by the experts, the following tables are required:

1) Expert list, including expert number, name, expert, teaching classes,

2) Curriculum including teacher number, course number, class and so on, for when rating an expert to choose the list of teachers and the corresponding class;

3) The scoring table, the scores include teacher name, courses, and other content, its role and student evaluation data sheets are basically the same. 


\section{Design of Evaluation Standardization}

In the practical application, a few students may make errors when operating. Both malicious and flattery score are also possible. Those will disturb normal evaluation. In addition, students of different classes evaluate different teachers with different scales in minds. Simple statistical summary is hardly convinced of its accuracy, reliability and comparability. Teaching evaluation scoring by a class of students complies the normal distribution.

We should take the original score of certain teacher in a class in such a way: Only take $90 \%$ data around standard deviation of the scores for valid data and remove the highest and lowest $10 \%$ abnormal data from the trusted zone. In practice, we should remove the highest $5 \%$ and lowest $5 \%$ and adjust valid number of students accordingly.

We should average scores by students to $m_{1}$ and average scores by experts to $m_{2}$, calculates the raw scores of teachers as follows:

$$
x=m_{1} q_{1}+m_{2} q_{2}, q_{1}, q_{2}>0, q_{1}+q_{2}=1
$$

where $q_{1}, q_{2}$ stands for weight of student evaluation and teacher evaluation respectively.

For example, $q_{1}=60 \%, q_{2}=40 \%$.

We should use the z-score method [16] (the standard method) to calculate standard scores. It is a number expressed as standard deviation scores in the group's relative position.

Score calculating formulae and steps:

$$
\bar{x}=\frac{1}{n} \sum_{i=1}^{n} x_{i}
$$

where $n$ is total number of teachers to be evaluated, $x_{i}$ is the raw score calculated for (1) for each teacher, in which student evaluation abnormal fluctuation is already handled;

$$
s=\sqrt{\frac{1}{n-1} \sum_{i=1}^{n}\left(x_{i}-\bar{x}\right)^{2}}
$$

where $S$ is the standard deviation of teacher score distribution[6];

$$
z_{i}=\frac{x_{i}-\bar{x}}{s}
$$

where $z_{i}$ is the z-score (the standard score);

$$
T_{i}=10 z_{i}+50
$$


This is the teacher's standard score in centesimal system. No matter what score system the original form may be in, the final result to be store into database will be in the same system to make analysis and comparison reasonable.

\section{Effect Analysis of Evaluation System}

Trial version of this system has been used in certain university. The result is expected. Evaluation data will dock with the data center, constantly collecting, archiving, data warehouse onto the data center, and will become increasingly perfect.

Implementation of evaluation system can reduce tedious data collection work by $70 \%$, save paper consuming, management fees and overtime paying by $90 \%$, increase working efficiency by 5 times. In the digital campus, students can use self-service terminal and information portal. We can achieve extremely high collection efficiency and authenticity. It is obviously superior to manual collection of stationery method.

Evaluation system is closely linked with the future of the university. Only qualified teaching makes a university develop at long term. It should become an integral part of the campus information system.

KEMAL TURHAN's system contains only teaching communication skills, presentation skills, and evaluation of four, each of which scored 1-5 points, there is no finer classification[7]; In our system there are experts in evaluation index system in six categories, each category is divided into a number of indicators, a total of 26 indexes weighted according to importance of division. The use of a scoring standard calculation method make the scheme more objective. Thus, we get better results in our system.

\section{Conclusions}

We presented the analysis and design of a teaching evaluation system which had been used in a university both on technique and theory. The result is favorable. We should relate student evaluation with new course selection and graduation management system to assure every student be serious to the evaluation. If a student does not do the evaluation seriously, he/she won't be allowed to take a new class or graduate. Thus, the evaluation will become more and more objective by time goes by. The system will become more and more perfect as time goes by. The research of evaluation system needs a continuous improvement.

\section{Acknowledgements}

The author is grateful to the editors, reviewers and any other helpful people for their valuable comments and suggestions to improve the presentation of this paper. This research was supported partly by the Scientific Project from Educational Bureau of Jiangxi Province (No. GJJ151019). Also, the author is grateful to the IJEME press for the free publication of this paper.

\section{References}

[1] Servlets with JavaBeans, http://stackoverflow.com/questions/14307633/servlets-with-javabeans.

[2] Computer Operating Systems: Unix vs Windows https://www.lifewire.com/operating-systems-unix-vs-windows-2180225, https://www.lifewire.com/operating-systems-unix-vs-windows-2180225.

[3] R.M. Gavilan, L.E. Bernold, Source Evaluation of Solid Waste in Building Construction, Journal of Construction Engineering \& Management, 120 (1994) 536-552.

[4] Course participants' evaluation of their knowledge and competencies, 2002.

[5] J.D. Morgan, D.J. Wright, H. Chrystyn, Pharmacoeconomic evaluation of a patient education letter aimed 
at reducing long-term prescribing of benzodiazepines, International Journal of Clinical Pharmacy, 24 (2002) 231-235.

[6] K. Hayes, A Lower Bound for the Most Deviant Z Score.Teaching Statistics, An International Journal for Teachers, 26: (2004).

[7] K. Turhan, F. Yaris, E. Nural, Does Instructor Evaluation by Students Using a Web-Based Questionnaire Impact Instructor Performance?, Advances in Health Sciences Education, 10 (2005) 5-13.

[8] N.S. THOMAIDIS, N. NIKITAKOS, G.D. DOUNIAS, THE EVALUATION OF INFORMATION TECHNOLOGY PROJECTS: A FUZZY MULTICRITERIA DECISION-MAKING APPROACH, International Journal of Information Technology \& Decision Making, 05 (2006) 89-122.

[9] L.J. Li Jihuai, Analysis on the evaluation of teachers ' teaching quality measurement system, Teaching and management, 3 (2006).

[10] W. Wachowiak, P.A. Balk, O. Savolainen, An Investigation of Online Course Management Systems in Higher Education: Platform Selection, Faculty Training, and Instructional Quality, International Journal of Information \& Communication Technology Education, 5 (2009) 117-132.

[11] M. Chi, K. Vanlehn, D. Litman, P. Jordan, An evaluation of pedagogical tutorial tactics for a natural language tutoring system: a reinforcement learning approach, International Journal of Artificial Intelligence in Education, 21 (2011) 83-113.

[12] S.H. Ahn, An evaluation of green manufacturing technologies based on research databases, International Journal of Precision Engineering and Manufacturing-Green Technology, 1 (2014) 5-9.

[13] The Java EE 5 Tutorial, http://docs.oracle.com/javaee/5/tutorial/doc/bnagy.html.

[14] Class SqlBean, http://www.docjava.com/book/cgij/jdoc/rdbms/SqlBean.html.

[15] Z.W. Liu Lin, Study on improving the efficiency of database access based on Java, Modern electronic technology, 223 (2006).

[16] What Does a Score Mean, http://www.personality-project.org/perproj/reliability.html.

\section{Authors' Profiles}

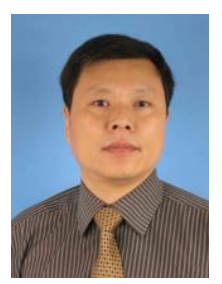

Ning Huang, born in 1958, received his Master's degree in applied mathematics and computer science from Jiangxi University, China in 1991, awarded senior engineer of the Industrial and Commercial Bank of China in 2001. He is now with Center of Modern Educational Technology, Gannan Normal University, Ganzhou, China, as an associate professor. His research interests include information security and digital campus.

How to cite this paper: Ning Huang,"Analysis and Design of University Teaching Evaluation System Based on JSP Platform", International Journal of Education and Management Engineering(IJEME), Vol.7, No.3, pp. 43-50, 2017.DOI: 10.5815/ijeme.2017.03.05 